BMJ Open

Diabetes

Research

\& Care

\section{Applications of physical performance measures to routine diabetes care for frailty prevention concept: fundamental data with grip strength, gait speed, timed chair stand speed, standing balance, and knee extension strength}

To cite: Yokoyama $\mathrm{H}$, Shiraiwa T, Takahara M, et al. Applications of physical performance measures to routine diabetes care for frailty prevention concept: fundamental data with grip strength, gait speed, timed chair stand speed, standing balance, and knee extension strength. BMJ Open Diab Res Care 2020;8:e001562. doi:10.1136/ bmjdrc-2020-001562

Received 14 May 2020 Revised 17 June 2020 Accepted 18 June 2020

\section{Check for updates}

(c) Author(s) (or their employer(s)) 2020. Re-use permitted under CC BY-NC. No commercial re-use. See rights and permissions. Published by BMJ.

For numbered affiliations see end of article.

Correspondence to Dr Hiroki Yokoyama; dryokoyama@yokoyamanaika. com

\section{ABSTRACT}

Introduction Progression of muscle strength weakening will lead to a poor physical performance and disability. While this is particularly important in patients with diabetes, the associations of reduced muscle strength measured by grip strength with clinical features and physical performance remain unclear. We investigated clinical features and physical performance measures in association with grip strength in elderly people with diabetes in a primary care setting.

Research design and methods A cross-sectional study was conducted enrolling 634 male and 323 female Japanese patients with type 2 diabetes aged 60 years or older. First, grip strength was measured and the associations of gender-specific grip strength with clinical features were evaluated. Second, in patients with a grip strength below the gender-specific median, physical performance measures, including gait speed, timed chair stand speed, knee extension strength, standing balance, and short physical performance battery scores, were investigated. Patients with and without a low performance defined by Asian Working Group for Sarcopenia were compared in terms of clinical features and physical performance measures.

Results Grip strength decreased according to aging and longer duration of diabetes and was independently related to body mass index, glycated hemoglobin A1C (HbA1c), serum albumin, albuminuria, neuropathy, and stroke in male patients, and to body mass index and albuminuria in female patients. The physical performance measures became worse proportionally to a decrease in the grip strength. Patients with a low performance exhibited a significantly older age, lower grip strength and serum albumin, higher albuminuria, and poorer physical performance measures than those without.

Conclusions Reduced grip strength was associated with glycemic exposure indicators of age-related duration, $\mathrm{HbA1C}$, and vascular complications. The physical performance measures became worse with

\section{Significance of this study}

What is already known about this subject?

- The number of elderly people with diabetes is increasing with the extension of life expectancy. Muscle strength weakening will progress with aging and lead to a low physical performance and to frailty. It is known that a decrease in muscle strength is accelerated by diabetes. However, the clinical features in association with muscle strength as measured by grip strength and physical performance measures remain unclear in the elderly people with diabetes.

What are the new findings?

- We revealed that reduced grip strength in elderly people with diabetes was associated with glycemic exposure indicators of age-related duration, glycated hemoglobin A1c and vascular complications, and the physical performance measures became worse proportionally to a decrease in the grip strength. Patients with a low performance exhibited a significantly older age, lower grip strength and serum albumin, higher albuminuria, and poorer physical performance measures than those without.

How might these results change the focus of research or clinical practice?

- The measurements of grip strength and physical performance in patients with diabetes may help promote intervention to prevent frailty in future studies. This study warrants the applications of the frailty concept to diabetes and applications of physical performance measures to the routine clinical practice.

decreasing grip strength. Measurements of grip strength and physical performance in patients with diabetes may help promote intervention to prevent frailty in future studies. 


\section{INTRODUCTION}

The number of elderly people with type 2 diabetes is markedly increasing with the extension of life expectancy in many countries, especially Japan. ${ }^{1-3}$ These patients are at a high risk for common geriatric syndromes. ${ }^{2-4}$ Also, they often have complications of several coexisting illnesses such as microvascular disease, hypertension, dyslipidemia, and cardiovascular disease (CVD). All these factors have significant effects to increase premature deaths and reduce the quality of life. ${ }^{3-5}$ Thus, to improve the prognosis in elderly patients with type 2 diabetes, it is necessary to fully understand the conditions and symptoms specific to the elderly and to consider appropriate diabetes management and preventive measures.

A series of epidemiological studies indicated that reduced muscle strength, commonly measured by grip strength, is associated with increased risks of mortality and CVD morbidity in the general population. ${ }^{6-10}$ Muscle strength of hand grip, elbow flexion, knee extension, and trunk flexion and extension measured in middle or old age were reported to be highly predictive of functional limitations and disability up to 25 years later. ${ }^{11}{ }^{12}$ Progression of muscle strength weakening will lead to a poor physical performance and eventually to disability. ${ }^{11-14}$ Thus, the prevention of disability and frailty conditions is important because these conditions severely reduce the quality of life of individuals and are economically burdening for public health. ${ }^{15-18}$

Diabetes per se was reported to be associated with a marked increase in the risks of physical disability and frailty. ${ }^{15} 16$ It is also known that a decrease in muscle strength is accelerated by aging, especially in people with diabetes compared with those without diabetes. ${ }^{131920}$ Thus, it is important to assess the muscle strength and physical performance in the clinical practice of diabetes care. ${ }^{4}$ In particular, elderly people with type 2 diabetes, if accompanied with a low grip strength, may have much higher risks of mortality and GVD morbidity.

However, in the clinical practice of diabetes care, especially in outpatient clinics, not only grip strength but also physical performances measures, which are practically applicable such as gait speed, timed chair stand speed, and knee extension strength, are not always performed routinely because of several practical issues such as limited consultation time, space, and manpower. The clinical features in association with grip strength and physical performance measures remain unclear in elderly people with type 2 diabetes. Therefore, the aim of this study was, using data measured as medical examinations for diabetes care: (1) to investigate the grip strength by age and the associations with clinical features in elderly Japanese people with type 2 diabetes and (2) to investigate physical performance measures in associations with clinical features and grip strength in those with a low grip strength.

\section{RESEARCH DESIGN AND METHODS}

\section{Study population}

All consecutive patients with type 2 diabetes aged 60 years or older who regularly visited the outpatient clinic of Jiyugaoka Internal Medicine in 2019 were included. The hand grip strength, as a strong indicator of muscle strength, was measured as one of the medical examinations for daily diabetes care. Using data of measured hand grip strength, this cross-sectional study was retrospectively performed with the aim of investigating hand grip strength in association with clinical characteristics and physical performance measures in a primary care setting. Reasons for exclusion included poor cognitive function (Mini-Mental State Examination <24); severe impairment of the liver, kidney, pulmonary, or cardiac function; or severe limitations in active daily life. Firstly, the data of grip strength were retrospectively collected, and the gender-specific mean values of grip strength according to the age stratification were calculated. Numerous papers reported that reduction of muscle strength will lead to a low physical performance. ${ }^{1-14}$ Therefore secondly, in patients with a grip strength below the gender-specific median, physical performance measures that are useful to identify the risk of frailty or falls ${ }^{21-27}$ were additionally measured as medical examinations for daily diabetes care, and the associations of grip strength with the physical performance measures were investigated to obtain fundamental data on physical performance measures in patients with weakened muscle strength. The study was approved by the local ethical committee and was carried out in accordance with the Declaration II of Helsinki.

\section{Measurements}

Type 2 diabetes was diagnosed according to the Japan Diabetes Society criteria, that is, fasting blood glucose $\geq 7.0 \mathrm{mmol} / \mathrm{L}$ or casual blood glucose $\geq 11.1 \mathrm{mmol} / \mathrm{L}$, plus glycated hemoglobin Alc (HbAlc) $\geq 6.5 \%$. The body mass index (BMI) was calculated as the ratio of body weight $(\mathrm{kg})$ to height squared $\left(\mathrm{m}^{2}\right)$. Blood pressure (BP) was measured in a sitting position after a 5 min rest. Non-fasting blood samples and random urine samples were obtained from each subject. HbA1c (normal range: $4.6 \%-6.2 \%$ ) levels were measured by high-performance liquid chromatography and presented as National Glycohemoglobin Standardization Program (\%) and International Federation of Clinical Chemistry (IFCC) values $(\mathrm{mmol} / \mathrm{mol})$. Serum concentrations of albumin and lipids were measured by OLYMPUS AU640 (Beckman Colter, Tokyo, Japan) and low-density lipoprotein cholesterol was calculated by Friedewald's formula. Serum and urinary concentrations of creatinine and urinary albumin were measured by enzymatic methods and turbidimetric immunoassay, respectively. The urinary albumin excretion rate was recorded as the albumin to creatinine ratio. The glomerular filtration rate (GFR) was estimated using the following equation by the Japanese Society of Nephrology: estimated GFR (eGFR) (mL/ 
$\left.\min / 1.73 \mathrm{~m}^{2}\right)=194 \times \mathrm{Scr}^{-1.094} \times \mathrm{Age}^{-0.287} \times 0.739$ (if female). Diabetic retinopathy was diagnosed after pupillary dilation by ophthalmologists. Neuropathy was diagnosed in patients with two or more of three components: presence of symptoms, absence of ankle tendon reflexes, and abnormal vibration perception threshold scores using a C128 tuning fork, where bilateral spontaneous pain, hypoesthesia or paresthesia of the legs was considered a neuropathic symptom. ${ }^{28}$ A previous medical history of macrovascular complications, that is, CVD, consisted of coronary artery disease (CAD), ischemic stroke, and peripheral artery disease (PAD). CAD included myocardial infarction, angina pectoris, and coronary interventions. Ischemic stroke included symptomatic brain infarction and carotid revascularization and did not include silent brain infarction, transient ischemic attack, or brain hemorrhage. PAD was diagnosed when intermittent claudication occurred, with the confirmation of an ankle-brachial pressure index $<0.9$ that was measured automatically by volume-plethysmographic apparatus or significant peripheral artery stenosis by angiography, or leg amputation above the ankle as a result of diabetes.

\section{Physical performance measures}

Hand grip strength was measured using Jamar Hand Dynamometer (T-2177; Toei Light, Saitama, Japan). Patients were encouraged to exert maximal grip strength. Two trials were conducted for each hand alternately, and the maximum value was used for the analyses. Walking speed was assessed by asking patients to walk at their usual pace over a $5 \mathrm{~m}$ course. Two walk times were recorded, and the faster of the two was used. To measure the sit-to-stand time, patients were asked to rise from a chair five times as fast as possible with verbal encouragement. Patients folded their arms across their chests while maintaining a $90^{\circ}$ upright posture with their feet shoulder-width apart and were instructed to stand up completely and make firm contact when sitting. The chair was of a standard height $(0.43 \mathrm{~m})$ without armrests. Two tests were performed and the faster one was recorded. Knee extension strength was measured using a handheld dynamometer ( $\mu$ Tas F-1; Anima, Tokyo, Japan). This method is new, portable, and simple, and was validated by comparing it with a conventional method using an isometric dynamometer. ${ }^{29}$ Furthermore, the usefulness for patients with diabetes was confirmed and the details of the measurements were as described by Nomura et al. ${ }^{30}$ In brief, patients maintained an upright posture with the hip and knee joints bent at $90^{\circ}$ in the end-sitting position. The sensor pad was applied on the anterior surface of the lower leg, which was $25 \mathrm{~cm}$ below the knee joint fissure. The test was performed primarily with the right leg three times, while the tester gave verbal encouragement. The maximal absolute value $(\mathrm{N}, 1 \mathrm{kgf}=9.8 \mathrm{~N})$ was used for the analyses. The intraclass correlation coefficients of the measurement were $\geq 0.9$.

The short performance physical battery (SPPB) score is based on timed measures of standing balance, gait speed and ability to rise from a chair. ${ }^{21-26}$ Each of the three performance measures was assigned a score from 0 to 4 , with 4 indicating the highest level of performance and 0 the inability to complete the test. A summary score (range: 0-12) was subsequently calculated by adding the three scores. For the balance test, patients were asked to maintain their feet in sideby-side, semitandem (heel of one foot beside the big toe of the other foot) and tandem (heel of one foot in front and touching the other foot) positions for $10 \mathrm{~s}$ each. Patients were assigned the following scores: 1 if they could only hold a side-by-side standing position for $10 \mathrm{~s} ; 2$ if they could only hold a semitandem position for $10 \mathrm{~s}$, but were unable to hold a full-tandem position for more than $2 \mathrm{~s} ; 3$ if they could only stand in a fulltandem position for 3 to $9 \mathrm{~s}$; 4 if they could only stand in a full-tandem position for $10 \mathrm{~s}$. Gait speed was scored as follows: $<0.46 \mathrm{~m} / \mathrm{s}=1 ; 0.47-0.64 \mathrm{~m} / \mathrm{s}=2 ; 0.65-0.82 \mathrm{~m} /$ $\mathrm{sec}=3 ; \geq 0.83 \mathrm{~m} / \mathrm{s}=4$. Five sit-to-stand time was scored as follows: unable $=0 ; \geq 16.7 \mathrm{~s}=1 ; 16.6-13.7 \mathrm{~s}=2 ; 11.2$ $13.6 \mathrm{~s}=3$; and $\leq 11.1 \mathrm{~s}=4$.

A low physical performance was defined according to the Asian Working Group for Sarcopenia (AWGS) definition by the presence of one of the following: gait speed $<1 \mathrm{~m} / \mathrm{s}$, five sit-to-stand time $\geq 12 \mathrm{~s}$, and SPPB score $\leq 9 .{ }^{31}$

\section{Statistical analysis}

Continuous data are expressed as the mean \pm SD if normally distributed and median (IQR) if nonnormally distributed. Grip strength was compared among patients stratified by every 5 -year age group, and the percentage of those with grip strength $<28 \mathrm{~kg}$ for males and $<18 \mathrm{~kg}$ for females, an AWGS cut-off value, is shown. To investigate the associations between grip strength and clinical features, patients were divided into four groups according to the quartiles of grip strength. For comparisons of continuous variables between two groups, unpaired Student's t-test for normally distributed variables or the Mann-Whitney $\mathrm{U}$ test for non-normally distributed variables was used. Comparisons of continuous variables among the four groups were performed by one-way analysis of variance. Non-normally distributed variables were first logarithmically transformed before any analyses. Comparisons between frequencies in the study groups were made by $\chi^{2}$ tests. A simple linear regression model was used to relate grip strength to other explanatory variables. Pearson's correlation coefficient to measure linear association between two quantitative variables and the coefficient of determination were calculated, and the fitted linear regression line was given. Multiple linear regression analysis to explore the multiple variables that may determine the grip strength was performed, in which continuous and categorical variables that were significant in the four-group analyses were employed. A p-value of $<5 \%$ (two-tailed) was considered significant. All analyses were performed using the statistical 
Table 1 Grip strength of patients with type 2 diabetes aged 60 years or older and the proportion of patients with grip strength $<28 \mathrm{~kg}$ for males and $<18 \mathrm{~kg}$ for females (ie, cut-off values by AWGS) according to the age stratification

\begin{tabular}{|c|c|c|c|c|c|c|}
\hline Age (years) & $60-64$ & $65-69$ & $70-74$ & $74-79$ & $80-$ & $P$ value \\
\hline $\mathrm{n}$ & 121 & 186 & 146 & 101 & 80 & \\
\hline$<28 \mathrm{~kg}(\%)$ & 3.3 & 10.2 & 19.9 & 29.7 & 56.3 & $<0.001$ \\
\hline \multicolumn{7}{|c|}{ Female patients with diabetes } \\
\hline Mean \pm SD $(\mathrm{kg})$ & $23.4 \pm 4.1$ & $21.1 \pm 3.9$ & $19.3 \pm 4.1$ & $18.7 \pm 3.6$ & $16.4 \pm 4.2$ & $<0.001$ \\
\hline$<18 \mathrm{~kg}(\%)$ & 4.4 & 20.6 & 36.7 & 34.8 & 64.6 & $<0.001$ \\
\hline
\end{tabular}

AWGS, Asian Working Group for Sarcopenia;

software package IBM SPSS statistics V.25 (SPSS Japan, Tokyo, Japan).

\section{RESULTS}

Table 1 shows mean values of grip strength and the percentage of grip strength $<28 \mathrm{~kg}$ for males or $<18 \mathrm{~kg}$ for females, gender-specific cut-off values suggested by AWGS, according to the age stratification. The mean values of grip strength were significantly decreased with aging, while the percentages of grip strength below the gender-specific cut-off values were significantly increased. Table 2 shows clinical characteristics of elderly patients with type 2 diabetes according to the genderspecific quartiles of grip strength. Male patients with a lower grip strength were significantly older, had a longer known duration of diabetes, higher values of HbAlc and albuminuria, and higher rates of retinopathy, neuropathy, and stroke, whereas they had lower BMI and serum albumin. There were no associations of grip strength with systolic BP, lipid profile, eGFR, CAD, or PAD. The results were similar in female patients, while HbA1c and stroke were not significantly different and eGFR was significantly different. In a subanalysis of patients without a prior history of CVD, the results of significant variables shown in table 2 were the same for both male and female patients. In multiple linear regression analysis where the significantly correlated factors in male patients were entered into the model, age $(\beta=-0.40$, $\mathrm{p}<0.0001)$, BMI $(\beta=0.35, \mathrm{p}<0.0001)$, HbA1c $(\beta=-0.68$, $\mathrm{p}<0.05)$, serum albumin $(\beta=2.76, p<0.01)$, albuminuria $(\beta=-0.67, p<0.01)$, neuropathy $(\beta=-1.21, p<0.05)$, and stroke $(\beta=-3.45, p<0.001)$ were independently related to the numerical values of grip strength (adjusted $R^{2}=0.33$, $\mathrm{F}$-value $=32.9, \mathrm{p}<0.001)$. The analysis in female patients indicated that age $(\beta=-0.27, p<0.0001)$, BMI $(\beta=0.16$, $\mathrm{p}<0.001)$, and albuminuria $(\beta=-0.18, \mathrm{p}<0.001)$ were independently related, whereas duration, serum albumin, eGFR, retinopathy, and neuropathy were not (adjusted $\mathrm{R}^{2}=0.26$, F-value $\left.=12.5, \mathrm{p}<0.001\right)$.

Physical performance measures were investigated in only those with grip strength below the median of $33.9 \mathrm{~kg}$ for males (253 available out of 300 patients, 84.3\%) and
$19.9 \mathrm{~kg}$ for females (122 out of 162 patients, 75.3\%). According to the gender-specific quartile of grip strength, male patients with a lower grip strength had a significantly slower gait speed, longer five sit-to-stand time, weaker knee extension strength, lower balance and SPPB scores, and higher percentage of a low physical performance defined by AWGS (table 3). In female patients with a lower grip strength, a similar trend was observed with less statistical power of significance because the number of available female patients for physical performance measures was small. Among the four groups of patients with grip strength below the median, there were no significant differences in clinical factors listed in table 1 besides the age for either males or females (data not shown). Figure 1 shows the relationships of grip strength with gait speed (A), five sitto-stand time (B), and knee extension strength (C), and the relationships of five sit-to-stand time with gait speed (D) and knee extension strength (E) in male and female patients with grip strength below the median. The grip strength was significantly correlated with the physical performance measures of gait speed, five sit-to-stand time, and knee extension strength, and five sit-to-stand time was significantly correlated with gait speed and knee extension strength.

Finally, clinical characteristics and physical performance measures were compared between those with and without a low physical performance, defined by AWGS, in patients with low grip strength (table 4). Male patients with a low performance $(\mathrm{n}=38,15.0 \%)$ demonstrated a significantly older age, lower grip strength, lower serum albumin, higher albuminuria and prevalence of stroke, and poorer physical performance measures. If limited to male patients with grip strength $<28 \mathrm{~kg}$, those with a low performance comprised $12.3 \%$ (12/97), and the statistical power became weak but showed a similar trend. Similar results in female patients, besides the stroke prevalence, were observed.

\section{DISCUSSION}

In elderly people with type 2 diabetes aged 60 years or older, grip strength, a strong indicator of muscle strength, was decreased with aging. The low grip strength was associated with old age, long duration of diabetes, 


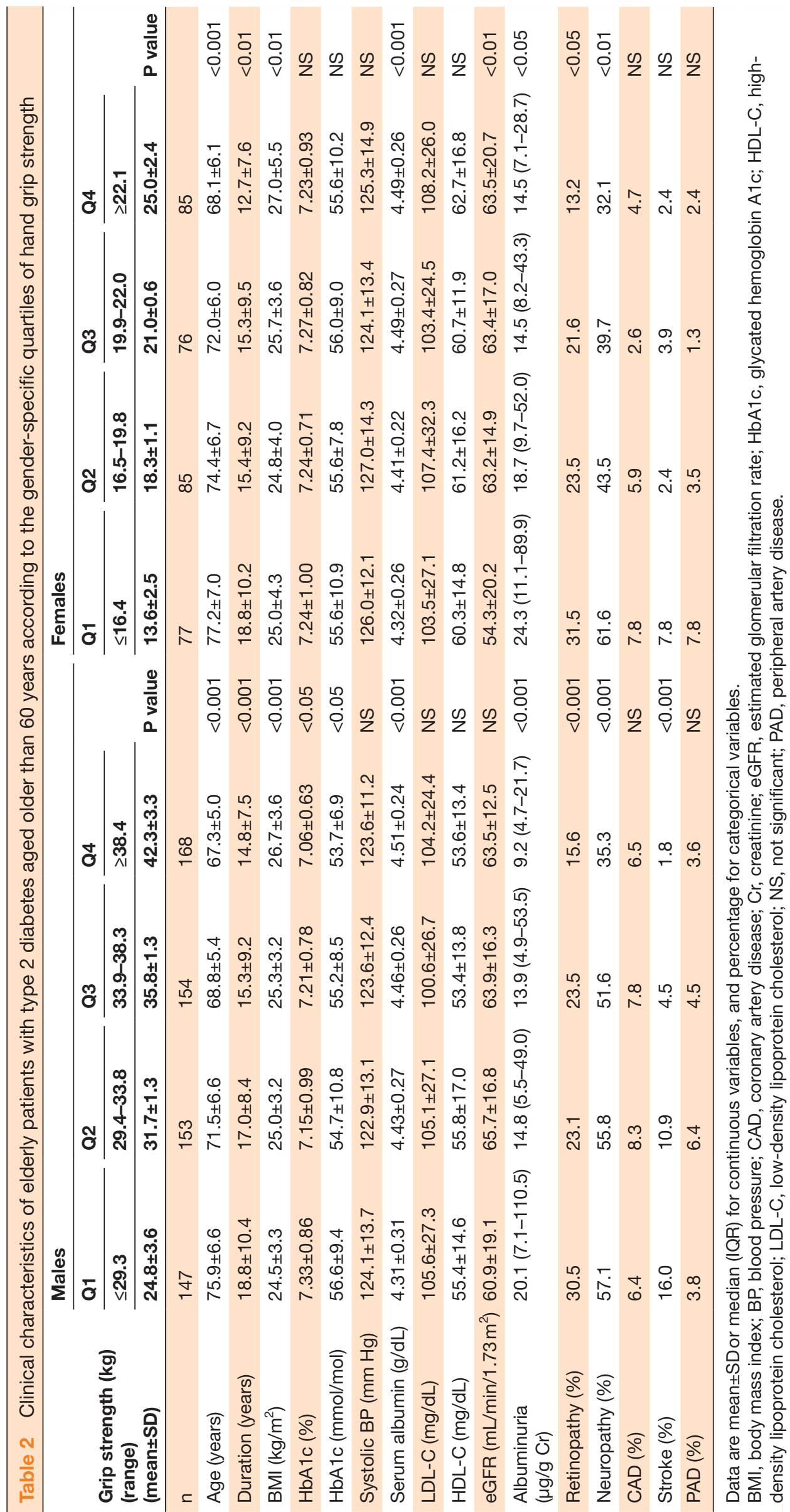

믕

ग 


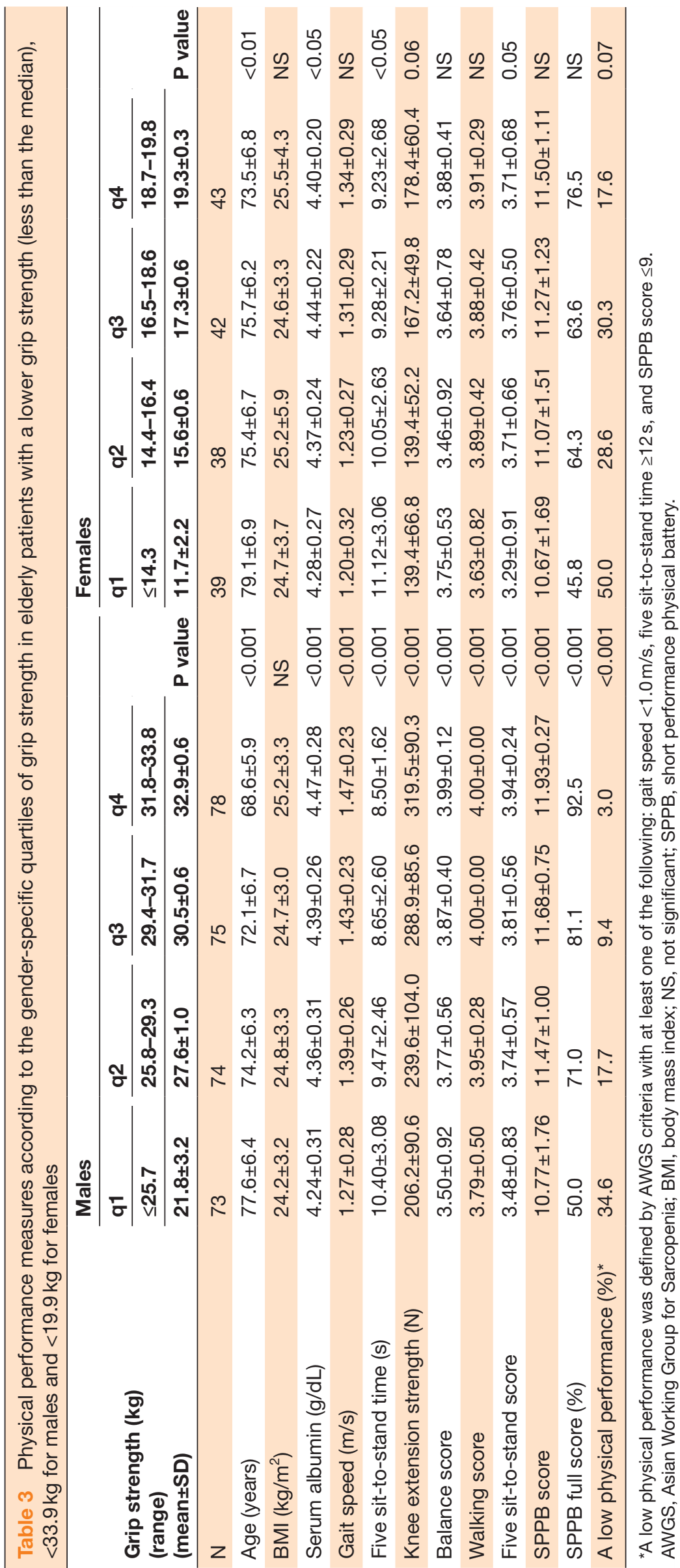

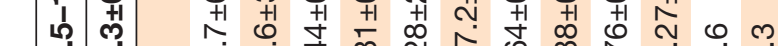


A

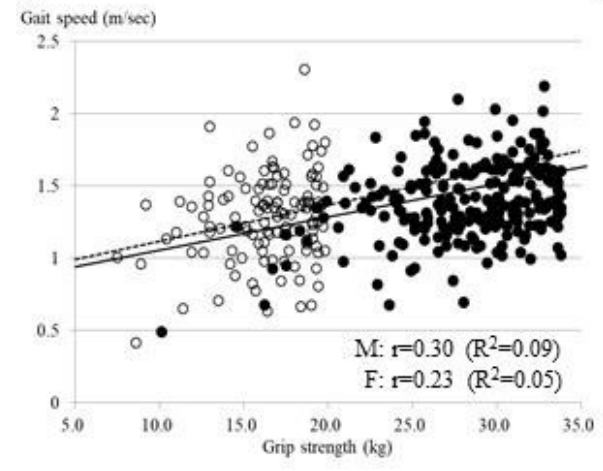

B

Five silto-stand time (sec)

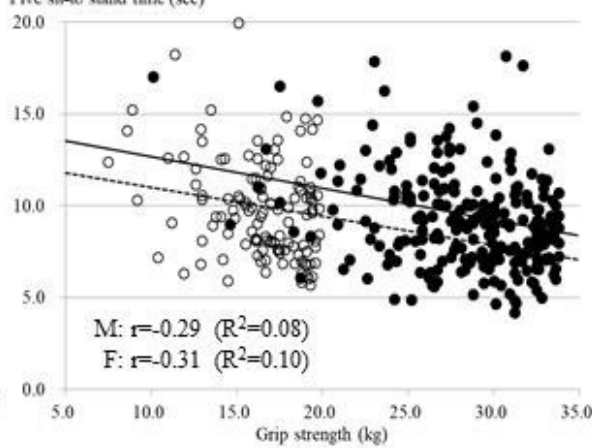

$\mathrm{C}$

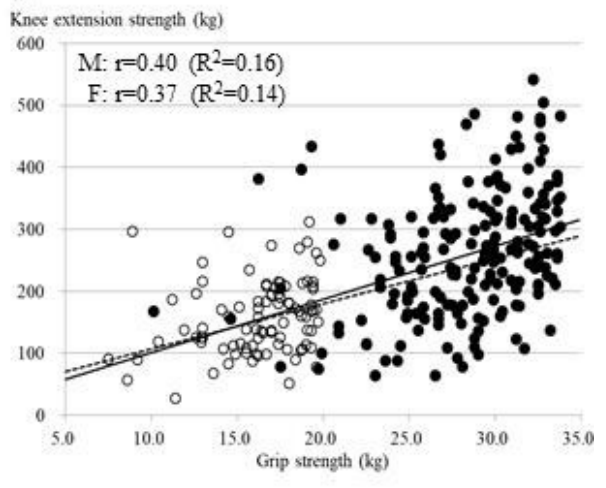

$\mathrm{E}$

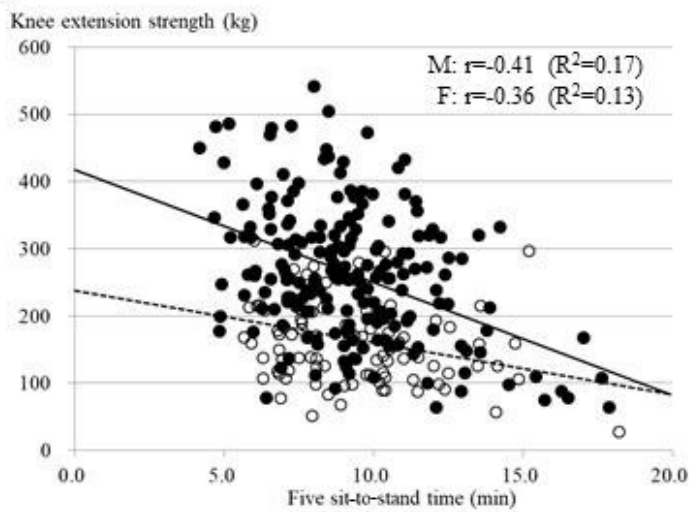

Figure 1 The upper panels show the relationships of grip strength with gait speed (A), five sit-to-stand time (B), and knee extension strength (C). The lower panels show the relationships of five sit-to-stand time with $5 \mathrm{~m}$ walking time (D) and knee extension strength (E). Closed and open circles indicate male (M) and female (F) patients, respectively. Solid and dotted lines indicate the fitted linear regression lines for male and female patients, respectively.

low BMI and serum albumin, high HbAlc and albuminuria, and presence of vascular complications. In addition, this study provided fundamental data on physical performance measures in patients with weakened muscle strength. Those with a low performance defined by AWGS were characterized by a significantly older age, lower grip strength and serum albumin, higher albuminuria and stroke, and poorer physical performance measures. While it requires future studies to investigate whether interventions to ameliorate physical performance measures could contribute to regaining muscle strength and prevention of disability and frailty, we believe that the relationship of grip strength with clinical features and physical performance measures indicated in this study may help monitor the effects of intervention.

This study showed that grip strength in elderly people with type 2 diabetes was decreased with aging. The grip strength in our diabetes population was apparently lower than that of a Japanese age-matched general population reported by Japanese governmental statistics e-Stat surveyed in 2017 (https://www.e-stat.go.jp/). Male patients with type 2 diabetes had a 4.4-5.2 kg lower grip strength in each age stratification than the general population. Female patients showed less grip strength as well. Of course, it is hard to directly compare the data of these two studies because, in the Japanese governmental statistics e-Stat, it is not opened how many patients with diabetes or other diseases were included and how the grip strength was measured. However, this trend that people with diabetes have lower grip power than general population is supported by the findings of reduced muscle strength in subjects with diabetes as compared with those without. ${ }^{13} 1920$

The association of reduced grip strength with high HbA1c, age-related long duration of diabetes, and vascular complications indicates that long-term glycemic exposure may affect not only the development of vascular complications but also muscle strength weakening, supported by previous studies. ${ }^{13} 161920$ Muscle strength weakening, or muscle catabolism, may be an important diabetes complication besides the conventional diabetic microvascular complications of neuropathy, retinopathy, and nephropathy. ${ }^{19}$ The absence of associations of grip strength with BP and lipid profiles, putative cardiovascular risk factors, was consistent with the findings from prospective follow-up studies, which indicated the predictive effect of grip strength on mortality and CVD morbidity independent of and beyond the effect of BP and lipids. ${ }^{6-9}$ Notably, patients with lower grip strength showed lower levels of serum albumin which is a nutritional marker. Several previous studies supported the associations of serum albumin with physical functional capacity, sarcopenia, and frailty. ${ }^{32-34}$ Taken together, these findings suggest that grip strength should be considered 
Table 4 Clinical characteristics and physical performance measures of those with and without a low performance in the elderly people with type 2 diabetes and grip strength $<33.9 \mathrm{~kg}$ for males and $<19.9 \mathrm{~kg}$ for females

\begin{tabular}{|c|c|c|c|c|c|c|}
\hline & \multicolumn{3}{|l|}{ Males } & \multicolumn{3}{|l|}{ Females } \\
\hline & Low performance & $\begin{array}{l}\text { Not low } \\
\text { performance }\end{array}$ & $P$ value & Low performance & $\begin{array}{l}\text { Not low } \\
\text { performance }\end{array}$ & $P$ value \\
\hline $\mathrm{n}$ & 38 & 215 & & 37 & 85 & \\
\hline Age (years) & $76.8 \pm 6.6$ & $73.3 \pm 6.6$ & $<0.01$ & $78.2 \pm 6.2$ & $73.5 \pm 6.5$ & $<0.001$ \\
\hline $\mathrm{BMI}\left(\mathrm{kg} / \mathrm{m}^{2}\right)$ & $24.6 \pm 3.0$ & $24.8 \pm 3.2$ & NS & $26.2 \pm 3.8$ & $24.4 \pm 3.9$ & NS \\
\hline Grip strength (kg) & $25.4 \pm 5.0$ & $29.2 \pm 3.8$ & $<0.001$ & $15.4 \pm 3.2$ & $16.9 \pm 2.4$ & $<0.01$ \\
\hline Serum albumin (g/dL) & $4.30 \pm 0.27$ & $4.42 \pm 0.28$ & $<0.05$ & $4.31 \pm 0.26$ & $4.43 \pm 0.21$ & $<0.01$ \\
\hline Albuminuria ( $\mu \mathrm{g} / \mathrm{g} \mathrm{Cr})$ & $37.2(8.1-244.6)$ & $15.5(6.1-48.5)$ & $<0.01$ & $37.2(8.1-244.6)$ & $15.5(6.1-48.5)$ & $<0.05$ \\
\hline Stroke (\%) & 26.3 & 8.4 & $<0.01$ & 10.8 & 4.7 & NS \\
\hline Gait speed $(\mathrm{m} / \mathrm{s})$ & $1.16 \pm 0.28$ & $1.44 \pm 0.23$ & $<0.001$ & $1.00 \pm 0.25$ & $1.41 \pm 0.24$ & $<0.001$ \\
\hline Five sit-to-stand time (s) & $13.29 \pm 2.48$ & $8.42 \pm 1.76$ & $<0.001$ & $12.55 \pm 2.69$ & $8.65 \pm 1.62$ & $<0.001$ \\
\hline $\begin{array}{l}\text { Knee extension strength } \\
\text { (n) }\end{array}$ & $190.7 \pm 92.0$ & $276.7 \pm 96.1$ & $<0.001$ & $131.4 \pm 56.8$ & $167.9 \pm 57.1$ & $<0.01$ \\
\hline Balance score & $3.24 \pm 1.05$ & $3.88 \pm 0.41$ & $<0.001$ & $3.35 \pm 1.01$ & $3.85 \pm 0.42$ & $<0.001$ \\
\hline Walking score & $3.63 \pm 0.63$ & $4.00 \pm 0.00$ & $<0.001$ & $3.49 \pm 0.80$ & $4.00 \pm 0.00$ & $<0.001$ \\
\hline Five sit-to-stand score & $2.68 \pm 0.87$ & $3.95 \pm 0.22$ & $<0.001$ & $2.89 \pm 0.84$ & $3.96 \pm 0.19$ & $<0.001$ \\
\hline SPPB score & $9.55 \pm 1.61$ & $11.83 \pm 0.47$ & $<0.001$ & $9.73 \pm 1.64$ & $11.81 \pm 0.48$ & $<0.001$ \\
\hline SPPB full score (\%) & 2.6 & 87.4 & $<0.001$ & 16.2 & 84.7 & $<0.001$ \\
\hline
\end{tabular}

Variables not significantly different between the two not shown, such as duration of diabetes, HbA1c, systolic BP, LDL-C, HDL-C, eGFR, retinopathy, neuropathy, CHD and PAD in males and females.

A low physical performance was defined by AWGS criteria with at least one of the following: gait speed $<1.0 \mathrm{~m} / \mathrm{s}$, five sit-to-stand time $\geq 12 \mathrm{~s}$, and SPPB score $\leq 9$.

AWGS, Asian Working Group for Sarcopenia; BMI, body mass index; BP, blood pressure; CHD, coronary heart disease; Cr, creatinine; eGFR, estimated glomerular filtration rate; HbA1c, glycated hemoglobin A1c; HDL-C, high-density lipoprotein cholesterol; LDL-C, low-density lipoprotein cholesterol; PAD, peripheral artery disease; SPPB, short performance physical battery.

for the prevention of CVD and frailty in the management of type 2 diabetes.

In particular, we found a strong association of albuminuria and diabetic neuropathy with grip strength and physical performance status in this study. This is the first study to demonstrate the association of grip strength with albuminuria in a large-scale investigation of elderly patients with type 2 diabetes. This is in line with the association of albuminuria with sarcopenia, a condition characterized by decreases in muscle mass, strength, and function, which was reported in previous studies including individuals without diabetes. ${ }^{35-37}$ Albuminuria reflects endothelial dysfunction and generalized vascular damage, ${ }^{38-40}$ which may affect muscle metabolism leading to reduced muscle strength. Impaired microcirculation, microinflammation, and oxidative stress within the muscle capillaries may be involved in this association. ${ }^{41}$ Diabetic neuropathy was weakly but independently associated with grip strength. The association between muscle strength and neuropathy was reported previously, ${ }^{19}{ }^{42}$ which is consistent with our findings. It remains uncertain how neurogenic-weakened muscle is involved.

Studies on old patients have indicated that physical capability, as assessed by physical performance measures such as shown in this study, was associated with sarcopenia, frailty, falls, and functional disability, and predicted mortality. ${ }^{6-13} 182743-45$ Usual walking speed, timed chair stand speed, grip strength, and standing balance inclusive of scoring SPPB are likely to be useful not only for evaluating the cross-sectional status but also for promoting physical activity intervention by monitoring the changes in the levels. ${ }^{43-46}$ Most of the studies dealing with physical performance measures examined individuals without diabetes, and only a few studies were done involving patients with diabetes. ${ }^{1345}$ These measures must be incorporated into daily practice for patients with diabetes.

Not surprisingly, the five measurements: grip strength, gait speed, timed chair stand speed, knee extension strength, and balance test were correlated with each other. These measurements are simple and inexpensive and may reflect different aspects of physical capability. For example, decreased knee extension strength in patients with diabetes as compared with individuals without diabetes was significantly correlated with a slow gait speed. ${ }^{13}$ The slow gait speed was accounted for more precisely if the statistical model included muscle density, quality, and ankle strength, because walking is a highly integrated function that requires the coordinated contribution of multiple physiological subsystems. ${ }^{47}$ The knee extension strength may reflect instant muscle power 
without weight-bearing, and speed of gait and chair stands may require more sophisticated coordination of muscle movement in the presence of weight-bearing. Although further complex tests including measurements of muscle endurance and flexibility will be useful for the full assessment of physical fitness, ${ }^{44}$ assessments of the five simple tests, how they correlated, dissociated, increased, or decreased, may be fundamental for future studies.

There are some limitations that must be addressed in this study. The test-retest reliability is always a problem for physical performance measures. Each test reflects multiple muscle strength and coordination of movement, and depends on a patient's response. However, the physical performance measures in this study are objective, portable, and quick, and were examined uniformly. The values in this study were obtained from repeated measurements, and the reproducibility was confirmed by us and others. ${ }^{29} 3048$ Namely, an interclass correlation coefficient of test-retest reliability on the measurements of the sit-to-stand time was 0.996 (95\% CI 0.988 to 0.999). Second, physical performance measures were not examined in patients with grip strength greater than the median. The tests are principally applicable to those with reduced muscle strength; therefore, they are not necessary for those with sufficient grip strength. Actually, in patients who underwent the tests, $92.5 \%$ of males in the highest quartile (q4 in table 3 ) achieved a full SPPB score, and this accounted for $76.5 \%$ of females. Third, data on muscle mass were not available in this study. With data on muscle mass, we can discriminate dynapenia from sarcopenia, both of which involve decreased muscle strength and should be measured in future studies. Finally, the study was performed in patients with diabetes who visited a single clinic, and so the findings should be confirmed by others. Also, a larger number of female patients may help evaluate gender differences.

In conclusion, muscle strength weakening based on low grip strength was associated with poor long-term diabetes management in terms of the age-related duration of diabetes, poor glycemic control, and vascular complications. In patients with weakened muscle strength, physical performance measures became worse proportionally to the decrease in grip strength. The relationship of grip strength with clinical features and physical performance measures indicated in this study may help monitor the effect of intervention on frailty prevention programs. We believe that applications of the frailty concept to diabetes and applications of physical performance measures to routine clinical practice are warranted.
${ }^{7}$ Graduate School of Comprehensive Human Sciences, University of Tsukuba, Tsukuba, Ibaraki, Japan

${ }^{8}$ Department of Internal Medicine, Niigata University Faculty of Medicine, Niigata, Japan

${ }^{9}$ Department of Medicine, Shiga University of Medical Science, Otsu, Shiga, Japan

Acknowledgements We thank Wakana Tanaka, Shun Annaka, and Shun Kakiuchi (Jiyugaoka Medical Clinic, Obihiro, Japan) for evaluating physical performance measurements and data collection, and Suguho Takahashi (Jiyugaoka Medical Clinic) for his skillful management of the data.

Contributors HY, TS, MT, MI, and NK designed the study. TN, MY, and HS provided valuable advice regarding physical performance measurements. HY generated data. HY and SA performed the statistical analysis. All authors contributed to the interpretation of the data. HY and SA performed drafting the manuscript. HY takes a responsibility for statistical method and organized for performing multivariate analysis. All authors critically reviewed the manuscript. $\mathrm{HY}$ is the guarantor of this work and as such had full access to all the data in the study and takes responsibility for the integrity of the data and the accuracy of the data analysis.

Funding The authors have not declared a specific grant for this research from any funding agency in the public, commercial or not-for-profit sectors.

Competing interests None declared.

Patient consent for publication Not required.

Ethics approval The study obtained ethics approval of the local ethical committee of Shiraiwa Medical Clinic (No: 190901) and participants gave informed consent before taking part.

Provenance and peer review Not commissioned; externally peer reviewed.

Data availability statement Data are available on reasonable request.

Open access This is an open access article distributed in accordance with the Creative Commons Attribution Non Commercial (CC BY-NC 4.0) license, which permits others to distribute, remix, adapt, build upon this work non-commercially, and license their derivative works on different terms, provided the original work is properly cited, appropriate credit is given, any changes made indicated, and the use is non-commercial. See: http://creativecommons.org/licenses/by-nc/4.0/.

ORCID iDs

Hiroki Yokoyama http://orcid.org/0000-0003-1602-4050

Hirohito Sone http://orcid.org/0000-0003-1263-2817

\section{REFERENCES}

1 NCD Risk Factor Collaboration (NCD-RisC). Worldwide trends in diabetes since 1980: a pooled analysis of 751 population-based studies with 4.4 million participants. Lancet 2016;387:1513-30.

2 Gregg EW, Cheng YJ, Srinivasan M, et al. Trends in cause-specific mortality among adults with and without diagnosed diabetes in the USA: an epidemiological analysis of linked national survey and vital statistics data. Lancet 2018;391:2430-40.

3 Nakamura J, Kamiya H, Haneda M, et al. Causes of death in Japanese patients with diabetes based on the results of a survey of 45,708 cases during 2001-2010: report of the Committee on causes of death in diabetes mellitus. J Diabetes Investig 2017;8:397-410.

4 Sinclair A, Morley J. Frailty and diabetes. Lancet 2013;382:1386-7.

5 Brownrigg JRW, Hughes CO, Burleigh D, et al. Microvascular disease and risk of cardiovascular events among individuals with type 2 diabetes: a population-level cohort study. Lancet Diabetes Endocrinol 2016;4:588-97.

6 Sasaki H, Kasagi F, Yamada M, et al. Grip strength predicts causespecific mortality in middle-aged and elderly persons. Am J Med 2007;120:337-42.

7 Leong DP, Teo KK, Rangarajan S, et al. Prognostic value of grip strength: findings from the prospective urban rural epidemiology (pure) study. Lancet 2015;386:266-73.

8 Kishimoto $\mathrm{H}$, Hata J, Ninomiya T, et al. Midlife and late-life handgrip strength and risk of cause-specific death in a general Japanese population: the Hisayama study. J Epidemiol Community Health 2014;68:663-8.

9 Celis-Morales CA, Welsh P, Lyall DM, et al. Associations of grip strength with cardiovascular, respiratory, and cancer outcomes and all cause mortality: prospective cohort study of half a million UK Biobank participants. BMJ 2018;361:k1651.

10 Rantanen T, Guralnik JM, Foley D, et al. Midlife hand grip strength as a predictor of old age disability. JAMA 1999;281:558-60. 
11 Rantanen T, Avlund K, Suominen H, et al. Muscle strength as a predictor of onset of ADL dependence in people aged 75 years. Aging Clin Exp Res 2002;14:10-15.

12 Visser M, Goodpaster BH, Kritchevsky SB, et al. Muscle mass, muscle strength, and muscle fat infiltration as predictors of incident mobility limitations in wellfunctioning older adults. J Gerontol Med Sci 2005;60A:324-33.

13 Volpato S, Bianchi L, Lauretani F, et al. Role of muscle mass and muscle quality in the association between diabetes and gait speed. Diabetes Care 2012;35:1672-9.

14 Sayer AA, Syddall H, Martin H, et al. The developmental origins of sarcopenia. J Nutr Health Aging 2008;12:427-32.

15 Wong E, Backholer K, Gearon E, et al. Diabetes and risk of physical disability in adults: a systematic review and meta-analysis. Lancet Diabetes Endocrinol 2013;1:106-14.

16 Aguayo GA, Hulman A, Vaillant MT, et al. Prospective Association Among Diabetes Diagnosis, $\mathrm{HbA}_{1 \mathrm{c}}$, Glycemia, and Frailty Trajectories in an Elderly Population. Diabetes Care 2019;42:1903-11.

17 Yamada M, Arai H. Social frailty predicts incident disability and mortality among community-dwelling Japanese older adults. J Am Med Dir Assoc 2018;19:1099-103.

18 Hanlon P, Nicholl BI, Jani BD, et al. Frailty and pre-frailty in middleaged and older adults and its association with multimorbidity and mortality: a prospective analysis of 493737 UK Biobank participants. Lancet Public Health 2018;3:e323-32.

19 Andersen $\mathrm{H}$, Nielsen S, Mogensen CE, et al. Muscle strength in type 2 diabetes. Diabetes 2004;53:1543-8.

20 Park SW, Goodpaster BH, Strotmeyer ES, et al. Accelerated loss of skeletal muscle strength in older adults with type 2 diabetes: the health, aging, and body composition study. Diabetes Care 2007;30:1507-12.

21 Guralnik JM, Ferrucci L, Simonsick EM, et al. Lower-Extremity function in persons over the age of 70 years as a predictor of subsequent disability. N Engl J Med 1995;332:556-62.

22 Guralnik JM, Simonsick EM, Ferrucci L, et al. A short physical performance battery assessing lower extremity function: association with self-reported disability and prediction of mortality and nursing home admission. J Gerontol 1994;49:M85-94.

23 Guralnik JM, Ferrucci L, Pieper CF, et al. Lower extremity function and subsequent disability: consistency across studies, predictive models, and value of gait speed alone compared with the short physical performance battery. J Gerontol A Biol Sci Med Sci 2000;55:M221-31

24 Vasunilashorn S, Coppin AK, Patel KV, et al. Use of the short physical performance battery score to predict loss of ability to walk 400 meters: analysis from the InCHIANTI study. J Gerontol A Biol Sci Med Sci 2009;64:223-9.

25 Minneci C, Mello AM, Mossello E, et al. Comparative study of four physical performance measures as predictors of death, incident disability, and falls in unselected older persons: the insufficienza cardiaca negli Anziani Residenti a Dicomano study. J Am Geriatr Soc 2015;63:136-41.

26 Lauretani F, Ticinesi A, Gionti L, et al. Short-Physical performance battery (SPPB) score is associated with falls in older outpatients. Aging Clin Exp Res 2019;31:1435-42.

27 Keevil VL, Luben R, Hayat S, et al. Physical capability predicts mortality in late mid-life as well as in old age: findings from a large British cohort study. Arch Gerontol Geriatr 2018;74:77-82.

28 Yasuda H, Sanada M, Kitada K, et al. Rationale and usefulness of newly devised abbreviated diagnostic criteria and staging for diabetic polyneuropathy. Diabetes Res Clin Pract 2007;77 Suppl 1:S178-83.

29 Katoh M, Hiiragi Y, Hirano M, et al. Isometric knee muscle strength measurement using a belt-stabilized hand-held dynamometer and an isokinetic dynamometer with and without trunk fixation: investigation of agreement of measurement values and factors influencing measurement. J Phys Ther Sci 2019;31:878-83.
30 Nomura T, Ishiguro T, Ohira M, et al. Isometric knee extension force in Japanese type 2 diabetic patients without apparent diabetic polyneuropathy: data from the multicenter survey of the isometric lower extremity strength in type 2 diabetes study. SAGE Open Med 2019;7:205031211882341-8.

31 Chen L-K, Woo J, Assantachai P, et al. Asian Working Group for Sarcopenia: 2019 consensus update on sarcopenia diagnosis and treatment. J Am Med Dir Assoc 2020;21:300-7.

32 Slee A, Birch D, Stokoe D. The relationship between malnutrition risk and clinical outcomes in a cohort of frail older hospital patients. Clin Nutr ESPEN 2016;15:57-62.

33 Uemura K, Doi T, Lee S, et al. Sarcopenia and low serum albumin level synergistically increase the risk of incident disability in older adults. J Am Med Dir Assoc 2019;20:90-3.

34 Chevalier S, Saoud F, Gray-Donald K, et al. The physical functional capacity of frail elderly persons undergoing ambulatory rehabilitation is related to their nutritional status. J Nutr Health Aging 2008;12:721-6.

35 Bůžková P, Barzilay JI, Fink HA, et al. Higher albumin:creatinine ratio and lower estimated glomerular filtration rate are potential risk factors for decline of physical performance in the elderly: the Cardiovascular Health Study. Clin Kidney J 2019;12:788-94.

36 Bouchi R, Fukuda T, Takeuchi T, et al. Sarcopenia is associated with incident albuminuria in patients with type 2 diabetes: a retrospective observational study. J Diabetes Investig 2017;8:783-7.

37 Han E, Lee Y-H, Kim G, et al. Sarcopenia is associated with albuminuria independently of hypertension and diabetes: KNHANES 2008-2011. Metabolism 2016;65:1531-40.

38 Feldt-Rasmussen B, Microalbuminuria F-RB. Microalbuminuria, endothelial dysfunction and cardiovascular risk. Diabetes Metab 2000;26 Suppl 4:64-6.

39 Stehouwer CD, Fischer HR, van Kuijk AW, et al. Endothelial dysfunction precedes development of microalbuminuria in IDDM. Diabetes 1995;44:561-4.

40 Yokoyama $\mathrm{H}$, Sone $\mathrm{H}$, Saito $\mathrm{K}$, et al. Flow-mediated dilation is associated with microalbuminuria independent of cardiovascular risk factors in type 2 diabetes - interrelations with arterial thickness and stiffness. J Atheroscler Thromb 2011;18:744-52.

41 Schmiedel O, Schroeter ML, Harvey JN. Microalbuminuria in type 2 diabetes indicates impaired microvascular vasomotion and perfusion. Am J Physiol Heart Circ Physiol 2007;293:H3424-31.

42 Nomura T, Ishiguro T, Ohira M, et al. Diabetic polyneuropathy is a risk factor for decline of lower extremity strength in patients with type 2 diabetes. J Diabetes Investig 2018;9:186-92.

43 Kwon S, Perera S, Pahor M, et al. What is a meaningful change in physical performance? findings from a clinical trial in older adults (the LIFE-P study). J Nutr Health Aging 2009;13:538-44.

44 Santos GOR, Wolf R, Silva MM, et al. Does exercise intensity increment in exergame promote changes in strength, functional capacity and perceptual parameters in pre-frail older women? A randomized controlled trial. Exp Gerontol 2019;116:25-30.

45 Rodriguez-Mañas L, Laosa O, Vellas B, et al. Effectiveness of a multimodal intervention in functionally impaired older people with type 2 diabetes mellitus. J Cachexia Sarcopenia Muscle 2019;10:721-33.

46 Perrotin S, Gilbert T, Dupuis M, et al. A multimodal and multidisciplinary program to prevent loss of mobility in patients aged over 70 years: study protocol of a multicenter cluster randomized study in primary care (the PRISME-3P study). BMC Geriatr 2019;19:48

47 Ferrucci L, Bandinelli S, Benvenuti E, et al. Subsystems contributing to the decline in ability to walk: bridging the gap between epidemiology and geriatric practice in the InCHIANTI study. $J A m$ Geriatr Soc 2000;48:1618-25.

48 Bohannon RW, Bubela DJ, Magasi SR, et al. Relative reliability of three objective tests of limb muscle strength. Isokinet Exerc Sci 2011;19:77-81. 